\title{
Editorial: Special Issue on Quantum Mechanical Modeling of Biological Systems
}

\author{
Jean-Philip PIQUEMAL ${ }^{1}$, Dennis SALAHUB ${ }^{2}$ \\ ${ }^{1}$ (Laboratoire de Chimie Théorique, UPMC Univ Paris 06, UMR 7616, Case Courrier 137, 4 Place Jussieu, F-75005 Paris, \\ France, jpp@lct.jussieu.fr) \\ ${ }^{2}$ (Department of Chemistry and Institute for Biocomplexity and Informatics, University of Calgary, 2500 University Drive \\ NW, Calgary, Alberta T2N 1N4, Canada, dsalahub@ucalgary.ca)
}

In 1944 Schrödinger published a remarkable little book called "What is Life?" (Cambridge University Press). In it, the father of the wave-function version of quantum mechanics ranged widely over some of the big scientific and philosophical questions of the day and, indeed, of today. On his way to questions of the nature of consciousness and self, he introduced the notion of the genetic material being an "aperiodic solid" in which mutations could be related to some sort of quantum jump between different forms of the matter within.

In 1944, and for many years thereafter, the question of the role of quantum mechanics in biology remained at the fringe. Schrödinger's aperiodic solid notion has now been borne out with the discovery of the structure of DNA and the myriad structures of DNA, RNA, proteins, lipids and their interactions that are now known. And, gradually over the years, the efficiency of quantum mechanical methodology and the power of computers have progressed to the point where models involving quantum mechanics for appropriate phenomena are emerging. Quantum mechanics is inexorably taking its place in the tool chest of biomolecular modeling, often combined with other methodologies.

This Special Issue of Interdisciplinary Science: Computational Life Sciences provides a snapshot of the state-ofthe-art of computational studies of biological systems and processes that involve quantum mechanics. We do not claim completeness; the field is too large and too diverse to be captured in a single volume. However, we think that the ten papers herein faithfully portray the excitement in the field, triumphs over some level of complexity, hurdles that still need to be overcome, and an indication of interesting and fruitful directions to follow.

Four of the papers involve quantum mechanics, working alone:

Ellinger and coworkers address the important problem of homochirality in the interstellar medium, a central question for astro-biology. Through extensive calculations of energies and dipole moments (important for detection by radio-astronomy) they propose target molecules for experimental detection: lactic acid $\left(\mathrm{HOCH}\left(\mathrm{CH}_{3}\right) \mathrm{COOH}\right.$, a precursor of alanine $\left(\mathrm{NH}_{2} \mathrm{CH}\left(\mathrm{CH}_{3}\right) \mathrm{OH}\right)$ and the simplest chiral molecule $\left(\mathrm{NH}_{2} \mathrm{CH}\left(\mathrm{CH}_{3}\right) \mathrm{OH}\right)$.

Cisneros has used a cluster model to study the dealkylation of DNA bases catalyzed by the AlkB family of enzymes. A catalytic iron atom is included in the model along with models of the nearby residues, succinate for the cofactor, and 1-methyl adenine, holding selected atoms fixed at their positions in the X-ray structure. Several mechanistic steps were examined and the results suggest that more than one possible mechanism may be operative, which is being followed up by more extensive, QM/MM models.

Russo and coworkers performed a comparative exploratory modeling of the binding region of beta-amyloid peptides with copper and zinc ions. They find that zinc has more conformational flexibility and they also compared specific interactions of copper and zinc with several important residues. Such basic information is a prerequisite for more elaborate models that will shed light on the structure and dynamics relevant to Alzheimer's and other prion diseases.

Piquemal and coworkers used broken-symmetry DFT and Spin-Flip TDDFT calculations to study a biomimetic model of Tyrosinase, containing a dioxygen molecule, two $\mathrm{Cu}(\mathrm{II})$ cations and six imidazole rings. An Electron Localization Function (ELF) analysis showed that the $\mathrm{Cu}-\mathrm{O}-\mathrm{Cu}$ three-center bonds exhibit very resilient out-of-plane density expansions that play a key role in docking, their 3D-orientation providing a topological signature of oxygen activation. Once again, the next steps will involve a QM/MM model for the environment, along with Free Energy 
Perturbation to include the solvent entropy.

Two papers include the effects of solvation using implicit models:

Zimmerman and Burda have explored the interactions of the anti-cancer drug Cisplatin with two sulfur-containing amino acids, cysteine and methionine. They examined variants of the Point-Charge Model and, in particular, developed a more realistic partial charge model for the cavity. The new methodology is applied to the calculation of reaction free energies of cisplatin with cysteine and methionine in solution at constant $\mathrm{pH}$.

In their contribution, Ryde and coworkers have looked in depth at the calculation of ligand-binding affinities, testing the influence of various implicit solvation models. They find that the accuracy depends strongly on the parameterization, but also on the magnitude of the solvation energy. If relative values are considered, all of the methods tested are accurate to $2-5 \mathrm{~kJ} / \mathrm{mol}$. They also mapped out the conformational dependence of QM charges and estimated the range of electrostatic interactions beyond a point-charge model. Multipoles up to octupoles and anisotropic polarizabilities have a significant influence for residues up to $10-15 \AA$ from the active site. They developed a new method PMISP (polarized multipole interactions with supermolecular pairs) with improved accuracy.

Three papers use QM/MM methodology to combine a quantum mechanical description of the central part of the system with a Molecular Mechanical description of the solvent and/or protein environment:

The effect of mutations on the fluorescence of Enhanced Cyan Fluorescent Protein was examined by Laurent and Assfeld, using a QM/MM model combined with a simple model to represent the electronic response of the surrounding medium by a polarizable continuum. The results reproduce the experimental trends and they are analyzed in terms of geometric deformations, electrostatic polarization and electronic response.

The CHARMM-deMon QM/MM interface was further developed by Noskov et al. who studied the hydration of three different monovalent cations, $\mathrm{Na}^{+}, \mathrm{K}^{+}$, and $\mathrm{Tl}^{+}$. MD simulations were performed with classical force fields, with a polarizable (Drude) force field and with the QM/MM approach. A new polarizable forcefield for $\mathrm{Tl}^{+}$was developed. All three levels of theory reproduce radial distribution functions accurately but important differences are observed in the probabilities of different coordination states if explicit account of polarization is included.

Moliner and coworkers have used QM/MM to study enzymatic pericyclic reactions. Traditionally, these reactions have been considered as a rarely exploited mechanism in cellular metabolism, despite their broad utility in the laboratory. Specifically, the chorismate to prephenate rearrangement has been scrutinized using a combination of semiempirical quantum chemistry and free energy profiles along the potential of mean force. The authors have recognized the inaccuracy of the semiempirical methods and propose two approaches for correction. In the first, a higher level of QM is used during the optimization, keeping the QM-MM interaction at the lower, semiempirical level. The second improved method combines information calculated at the lower level with selected higher level results for a reduced number of geometries.

Finally, one paper goes beyond the usual Born-Oppenheimer approximation and includes quantum effects for the nuclear motion as well as for the electronic structure:

Ranaghan and Mullholland present a review of recent computational studies of quantum tunneling in enzyme reactions, with a focus on soybean lipoxygenase, dihydrofolate reductase, methyl amine dehydrogenase and aromatic amine dehydrogenase. Proton, hydride and hydrogen atom transfers are considered and kinetic isotope effects are the prime target. A variety of approaches have been used (Variational Transition State Theory, mixed quantumclassical methods, Feynman's path integral methodology, sometimes with the centroid dynamics approximation, etc...). The paper provides an excellent review of QM and QM/MM calculations involving hydrogen transfers, with quantum mechanics working on the electrons and also on the proton dynamics.

While a complete answer to Schrödinger's question "What is Life" must still lie far in the future, it seems clear to us that quantum mechanics has an essential role to play in seeking further insight. QM will, in the fullness of time, be incorporated into a multiscale conceptual framework and new computational methodologies will be developed to model life processes of ever increasing complexity and with ever increasing fidelity. We think the 10 papers of this Special Issue will prove to be of lasting value; they are steps along the way ... 